\title{
Liquid crystal Fresnel zone lens based on single-side-patterned photoalignment layer
}

\author{
X. Q. Wang, F. Fan, T. Du, A. M. W. Tam, Y. Ma, A. K. Srivastava,* \\ V. G. Chigrinov, and H. S. Kwok \\ Partner State Key Laboratory on Advanced Displays and Optoelectronics Technologies, \\ Hong Kong University of Science and Technology, Hong Kong, China \\ *Corresponding author: abhishek_srivastava_lu@yahoo.co.in
}

Received 3 December 2013; revised 15 February 2014; accepted 16 February 2014; posted 19 February 2014 (Doc. ID 202338); published 25 March 2014

\begin{abstract}
In this article, we disclose a method to fabricate a liquid crystal (LC) Fresnel zone lens (FZL) with high efficiency. The LCFZL, based on patterned planar-aligned regions, has been prepared by means of a twostep photoalignment technique. The proposed binary-phase LCFZL manifests 39\% diffraction efficiency at the focal point, which is close to the theoretical limit, $41 \%$. Moreover, because of a lower driving voltage and faster response time, these elements could find application in many modern devices. (C) 2014 Optical Society of America

OCIS codes: (220.0220) Optical design and fabrication; (230.0230) Optical devices; (050.1380) Binary optics; (050.1965) Diffractive lenses; (050.1970) Diffractive optics; (260.1440) Birefringence. http://dx.doi.org/10.1364/AO.53.002026
\end{abstract}

\section{Introduction}

A fast switchable Fresnel lens with high efficiency is highly desirable for verity of applications and therefore has been well documented in recent years [1-7]. There are two most common approaches to fabricate a liquid crystal (LC) Fresnel zone lens (FZL) (LCFZL). The first approach exploits striped electrodes to generate periodic electrical field distribution and locally control the directors of uniformly aligned LCs [1]. The second approach deals with directly guiding the initial LC director to realize periodic refractive index distribution profile through patterned polymer relief [ㅇ,9] polymer dispersed LCs [10,11], polymerstabilized LCs [12-15], dye-doped LCs [16-19], and UV-modified photoalignment film $[4,5]$ etc.

Recently, Lu et al. have proposed $2 \mathrm{D} / 3 \mathrm{D}$ switchable display based on a switchable Fresnel lens by deploying the patterned electrode [1]. The optical quality is acceptable; however, the switching time of the proposed LCFZL is $0.35 \mathrm{~s}$. In another approach,

$1559-128 \mathrm{X} / 14 / 102026-04 \$ 15.00 / 0$

(C) 2014 Optical Society of America
FZL is fabricated by the modification of the LC alignment based on UV exposure. The fabrication is complicated and requires high exposure energies to generate the FZL profile; moreover, the switching off time is $\sim 180 \mathrm{~ms}$, which is very large [5].

In 2010, Lou et al. proposed relatively simple fabrication of LCFZL based on a nanoimprinting technique. A Ni shim stamp has been used as a master stamp, and the FZL profile has been created on a UV curable prepolymer film by UV imprinting [9]. This approach is relatively simpler but has several issues with the master stamp and the response time of the LCFZL.

Recently, we proposed an LCFZL based on alternatively distributed twisted nematic (TN) and planaraligned (PA) alignment domains [7]. The diffraction efficiency of such a device can reach up to $\sim 20 \%$ when the twisted angle is $90^{\circ}$. However, due to the zone plate concept, it can only provide the amplitude modulation, and therefore the diffraction efficiency of such device is much less than that of a binaryphase LCFZL ( 41\%).

Most of these systems are characterized by the low efficiency, rather complicated fabrication procedure 
and extremely large switching time (typically few hundreds of millisecond). On the other hand, photoalignment technology provides us an opportunity for easy fabrication of such FZL structure, which is characterized by high efficiency and considerable small response time.

Lin et al. have disclosed a polarizationindependent LCFZL based on double-side-patterned photoalignment layers [16]. Such FZL are characterized by high diffractive efficiency of $37 \%$ and the total switching time, for a $3 \mu \mathrm{m}$ thick cell, of $26 \mathrm{~ms}$. The technology is based on the UV-guided dye-doped LC cell. The dye-doped LCs are known to have several issues regarding compatibility and chemical and photo stability of the dye in the LC bulk [20]. Furthermore, the matching of the FZL pattern on the top and bottom substrate of the FLZ cell is another big issue that demands a highly collimated pumping beam.

In this article, we disclose an LCFZL based on a single-side-patterned planar-aligned domain while the other substrate is only washed in isopropanol. Such binary-phase LCFZLs are characterized by a response time of $\sim 3.8 \mathrm{~ms}$, driving voltage less than $6.5 \mathrm{~V} / \mu \mathrm{m}$ [7]. Its diffraction efficiency can theoretically reach up to $\sim 41 \%$; moreover, it is polarizationindependent.

\section{Methodology}

Figure 1(a) shows the schematic configuration of LC in the cross section. The orientation of the LC molecules under the white and black regions is orthogonal to each other. Furthermore, because of optically active alignment layer, such LCFZL can be tuned electrically and optically as well. The optical tunability is discussed later in this article. The proposed design of the LCFZL cell consists of two substrates, one of which has an optically active photoalignment layer while the other substrate is a bare ITO glass plate. A sulfonic azo dye (SD1) (Dai-Nippon Ink and Chemicals, Japan) is used as an optically active

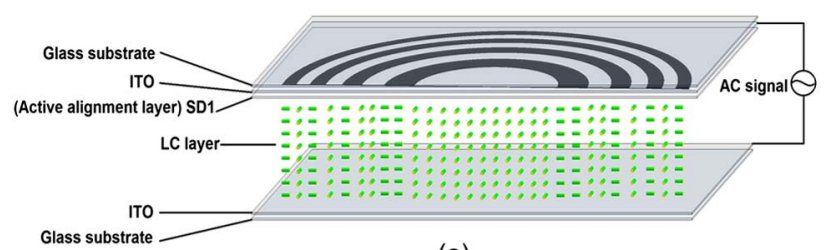

(a)

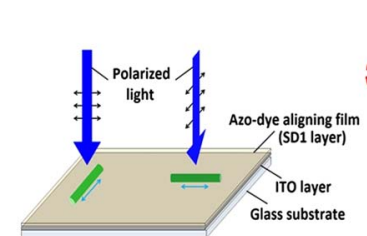

(b)

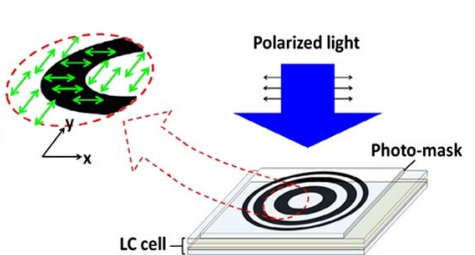

(c)
Fig. 1. Configuration and fabrication of FZL. (a) Configuration of FZL. (b) Schematics of photoalignment. (c) Patterned photoalignment process. The zoomed area in the red dotted circle shows the easy axis distribution in the two alignment domains. alignment layer. When the SD1 layer is exposed through polarized light, the energy absorbed by SD1 molecules is proportional to the square of the cosine $\theta$; here $\theta$ is an angle between the polarization plane of light and Azo dye absorption oscillator (chromophore) [21] and provides an excess of chromophores in a direction perpendicular to the plane of the polarization of the pumping light [21]. Thus the exposure of the SD1 substrate by the polarized light of wavelength $450 \mathrm{~nm}$ provides the alignment in the direction perpendicular to the plane of polarization of the impinging light with almost zero pretilt angle and high anchoring energy [see Fig. 1(b)] $[21,22]$. Thus the LCFZL cell has been fabricated by two ITO (indium-tin-oxide)-coated glass substrates, one of which has been coated with SD1 (1 wt. \% in $\mathrm{N}, \mathrm{N}$-dimethylformamide); after which two glass substrates were assembled to fabricate an LC cell with $1.5 \mu \mathrm{m}$ spacers sandwiched between these substrates. The cell thickness was measured to be $1.5 \pm$ $0.1 \mu \mathrm{m}$ by interferometry. The cell was initially exposed with a linearly polarized writing beam of $\lambda_{w}=450 \pm 10 \mathrm{~nm}$ for a dose of $5 \mathrm{~J} / \mathrm{cm}^{2}$. Afterward, the same cell was turned $90^{\circ}$ and exposed again through a Fresnel-zone-patterned photo-mask (the pattern was designed according to the equation $R_{k}^{2}=k \lambda f, k=1,2,3 \ldots$, where $R_{k}$ is the radius of the Fresnel zone, $\lambda$ is the wavelength of $632.8 \mathrm{~nm}$, and $f$ is the designed focal length), which was produced by photolithography and was in proximity contact with the cell [see Fig. 1(c)]. The SD1 molecules in the exposed window areas were realigned to have the easy axis orthogonal to the original orientation of those in mask shadow regions. Thus the fabricated cell shows the FZL profile, and thereafter the LC MLC-6080 (from Merck) was injected into the cell by the capillary action. This LC with $\delta n=n_{e}-n_{o} \approx 0.2$ meets the half-wave condition, $d=(\lambda / 2 \delta n)$, for $d=1.5 \mu \mathrm{m}$ is the cell thickness, $\lambda \approx 600 \mathrm{~nm}$ is the wavelength, $\delta n$ is the birefringence of the LC.

In the absence of the electric field, the light passes through the two different alignment domains of the LCFZL cell, and it results in redistribution of energy of the outward beam and generates the Fresnel pattern. Whereas, in the presence of the electric field, all LC molecules in both domains switch to the vertical position; thus the FZL profile on the $\mathrm{LC}$ cell vanishes. Thus the switchable LCFZL cell was achieved with the application of an electric field with an arbitrary waveform.

Because the alignments of LC in two domains are orthogonal to each other, and the LC in every two adjacent domains provides $\pi$ phase shift, the diffraction efficiency of such LCFZL is $41 \%$, no matter whether the polarization of the incident light is along the easy axis of the LC in odd zones or even zones. Light with any polarization state can be expressed as the superposition of two linearly polarized lights with polarization orthogonal to each other. Therefore, the proposed LCFZL is polarization-independent [23]. 


\section{Experiment and Results}

Figure 2(a) represents the optical microphotograph of the LCFZL (lens I) with the focal length of $40 \mathrm{~cm}$ under two crossed polarizers. The arrows in the first and second zones indicate the orientation of LC in odd zones and even zones, which is parallel to the polarizer (P) and analyzer (A), respectively. Figure 2(b) represents the optical micrograph of the LCFZL (lens II) with the focal length of $20 \mathrm{~cm}$ under two crossed polarizers. The arrows also indicate the direction of easy axis of LC in each zone. The white circles in both figures represent the boundaries between the odd zones and even zones

The He-Ne laser with a wavelength of $632.8 \mathrm{~nm}$ was used to study different features of fabricated LCFZL. The laser beam was magnified 10 times by a beam expander. A screen was placed at a distance of $\sim 30 \mathrm{~cm}$ from the LCFZL, and a digital SLR camera (Canon EOS 600D) was used to capture the images. Afterward, the image was analyzed by MATLAB.

For the theoretical elaboration, we assume that the polarization of the incident light is along the easy axis ( $x$ axis) of LC in odd zones. The electric field of the incident light is $\boldsymbol{E}_{\mathrm{in}}$, with its linear polarization parallel to $x$ axis in the $x y$ coordinate system, is given by $E_{\text {in }}=\left[\begin{array}{c}E_{0} \\ 0\end{array}\right]$. The outgoing field $E_{\text {out }}$ through the odd zones and even zones of the LC zone lens in the $x y$ coordinate system is given by

$$
E_{\text {out } \mid \text { odd }}=\left[\begin{array}{c}
E_{0} e^{\operatorname{in}_{e} \frac{2 \pi}{\lambda} d} \\
0
\end{array}\right] \quad \text { and } \quad E_{\text {out } \mid \text { even }}=\left[\begin{array}{c}
E_{0} e^{\mathrm{in}_{0} \frac{2 \pi}{\lambda} d} \\
0
\end{array}\right]
$$

where $n_{e}$ and $n_{o}$ are the refractive indices for long axis and short axis of the $\mathrm{LC}$, respectively, $\lambda$ is the wavelength and $d$ is the thickness of the LC cell. The first-order diffracted light field $D_{1}$ is given by

$$
D_{1}=\frac{\int_{0}^{2 A_{1}} E_{\mathrm{out}} e^{-i 2 \pi\left(A / 2 A_{1}\right)} d A}{2 A_{1}}
$$

where $A_{1}$ denotes the area of the first odd zone of the zone lens. Therefore, the theoretical diffraction efficiency of the first-order diffracted beams is $\eta_{1}=$ $\left(\left|D_{1}\right|^{2} /\left|E_{\text {in }}\right|^{2}\right)=[(\sin (\pi / 2) / \pi / 2)]^{2}=\left(4 / \pi^{2}\right)[\underline{16}]$.

In the experiment, the light intensity $I_{1}$ of the firstorder diffracted light was measured at the primary focal point by subtracting the zeroth-order light

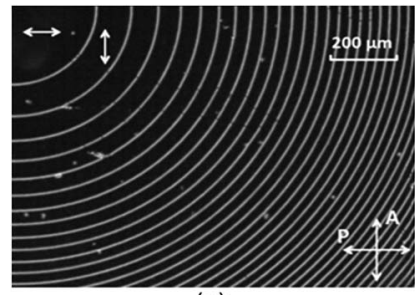

(a)

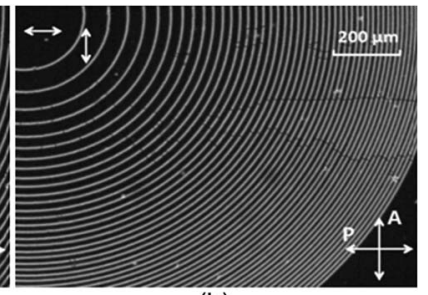

(b)
Fig. 2. Optical microphotograph of LCFZL with the focal length of (a) $40 \mathrm{~cm}$ and (b) $20 \mathrm{~cm}$ under two crossed polarizers. intensity in the center of the diffraction pattern. After the incident light intensity $I_{0}$ was measured, the diffraction efficiency $\eta=\left(I_{1} / I_{0}\right)$ of $\sim 39 \%$ was obtained, which is close to the theoretical limit [24]. The experimental errors and deviation form the half-wave conditions, which can be attributed to the slight disagreement in the experiment and theory.

Figure 3 shows the diffraction profile of the proposed structure in diffractive state $3(\mathrm{a})$ of lens I (focal length $40 \mathrm{~cm}$ ), and nondiffractive state $3(\mathrm{~b})$. When the electric field of $U \gg$ threshold voltage is applied, the LC in the odd zones and even zones represents the same optical state, of which the LC molecules tend to be vertically aligned; therefore, at high electric field $(U>8 \mathrm{~V})$, the diffraction pattern completely disappears.

Figure 3(c) shows the experiment setup for capturing the projection image of the LCFZL (lens I). A transparent letter $\mathrm{S}$ is placed between the beam expander and the LC cell. A screen is placed at a distance of $30 \mathrm{~cm}$ to the LCFZL cell. Figure 3(d) shows the projection image in nondiffractive state of the proposed LCFZL, and Fig. 3(e) shows the magnified projection image in its diffractive state.

The on-time and off-time of our proposed FZL are dependent on the response times of the ECB cell. The

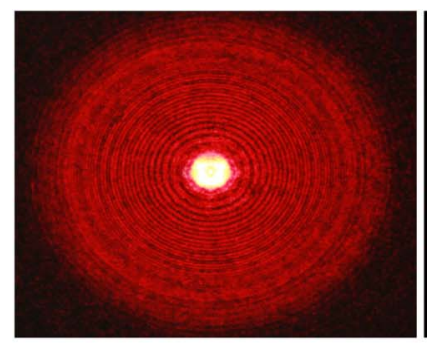

(a)

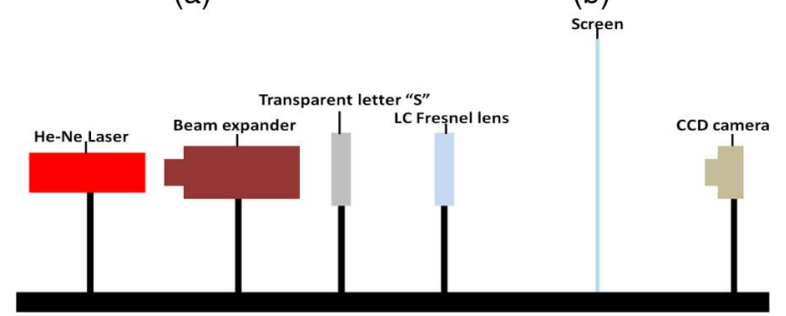

(c)

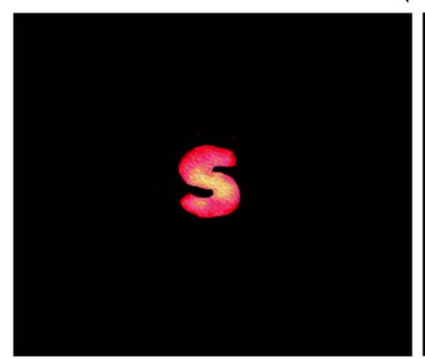

(d)

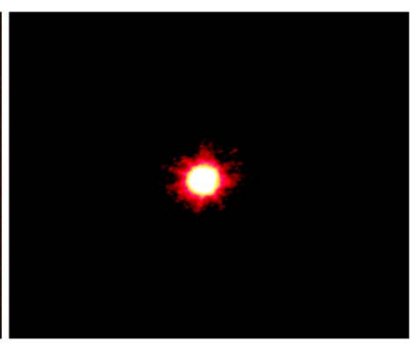

(b)

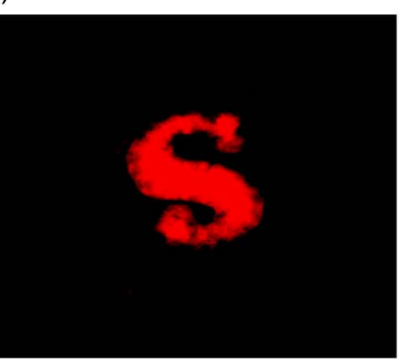

(e)
Fig. 3. Diffraction profiles and projection images. (a) Diffraction pattern in diffractive state. (b) Profile in nondiffractive state. (c) Setup for capturing the projection image. (d) Projection image in nondiffractive state. (e) Projection image in diffractive state. 
measurement system was the same as that used in our previous experiment $[7,23]$. The experimental results of the LC cell (lens II) are $\tau_{\text {on }} \approx 400 \mu \mathrm{s}$ and $\tau_{\text {off }} \approx 3.4 \mathrm{~ms}$, and the total response time is $3.8 \mathrm{~ms}$, which is relatively faster than the existing devices and can be further optimized by the selection of proper cell thickness and viscosity of the LC.

Moreover, the focal length of the proposed LCFZL cell can be erased and rewritten for the FZL pattern with different focal length by using a polarized laser $\left(\lambda_{w}=450 \pm 10 \mathrm{~nm}\right)$ [22]. The speed of the erasing and rewriting is the same and depends on the anchoring energy of the alignment layer and elastic parameters of the LC [22]. The anchoring energy of the optically active alignment layer in the proposed LCFZL cell can be tuned by doses of the exposing energy. Therefore, a high-power laser and proper selection of the elastic parameter of the LC provides a response time of less than $100 \mathrm{~ms}$ [22].

\section{Conclusion}

In this article, we have demonstrated a switchable LCFZL with the efficiency of $\sim 39 \%$ and response time of $\sim 3.8 \mathrm{~ms}$. As compared with the existing devices, the proposed LCFZL is relatively faster and a less power-consuming device. The photoalignment on only one substrate of the cell makes the fabrication process extremely simple. Moreover, the blue polarized laser can also tune the focal length of the proposed LCFZL cell if needed. The response time for optically tuning the focal length depends on the elastic parameters of $\mathrm{LC}$ and the power of the laser, which can be less than $100 \mathrm{~ms}$ [22]. Thus, with the features of fast switching, higher efficiency, and easy fabrication, these elements could find application in many modern optical and electro-optical devices.

We gracefully acknowledge support from the State Key Lab on Advanced Displays and Optoelectronics Technologies, HKUST.

\section{References}

1. J. G. Lu, X. F. Sun, Y. Song, and H. P. D. Shieh, "2-D/3-D switchable display by Fresnel-type LC lens," J. Disp. Technol. 7, 215-219 (2011).

2. C. Dorrer, S. K. H. Wei, P. Leung, M. Vargas, K. Wegman, J. Boulé, Z. Zhao, K. L. Marshall, and S. H. Chen, "High-damagethreshold static laser beam shaping using optically patterned liquid-crystal devices," Opt. Lett. 36, 4035-4037 (2011).

3. X. Q. Wang, F. Fan, J. T. Sun, L. Wang, A. Srivastava, and V. G. Chigrinov, "Evaluation of LC Fresnel phase plate utilized as colour filter," Mol. Cryst. Liq. Cryst. 559, 228-240 (2012).

4. S. J. Hwang, T. A. Chen, K. R. Lin, and S. C. Jeng, "Ultravioletlight-treated polyimide alignment layers for polarizationindependent liquid crystal Fresnel lenses," Appl. Phys. B 107, 151-155 (2012).

5. S. C. Jeng, S. J. Hwang, J. S. Horng, and K. R. Lin, "Electrically switchable liquid crystal Fresnel lens using
UV-modified alignment film," Opt. Express 18, 26325-26331 (2010).

6. M. S. Millán, E. Pérez-Cabré, and J. Otón, "Multiplexing schemes for an achromatic programmable diffractive lens," J. Phys. 139, 012016 (2008).

7. X. Q. Wang, A. K. Srivastava, V. G. Chigrinov, and H. S. Kwok, "Switchable Fresnel lens based on micropatterned alignment," Opt. Lett. 38, 1775-1777 (2013).

8. C. R. Lee, K. C. Lo, and T. S. Mo, "Electrically switchable Fresnel lens based on a liquid crystal film with a polymer relief pattern,” Jpn. J. Appl. Phys. 46, 4144-4147 (2007).

9. Y. M. Lou, Q. K. Liu, H. Wang, Y. C. Shi, and S. L. He, "Rapid fabrication of an electrically switchable liquid crystal Fresnel zone lens," Appl. Opt. 49, 4995-5000 (2010).

10. H. Jashnsaz, N. H. Nataj, E. Mohajerani, and A. Khabbazi, "All-optical switchable holographic Fresnel lens based on azo-dye-doped polymer-dispersed liquid crystals," Appl. Opt. 50, 4295-4301 (2011).

11. H. Ren, Y. H. Fan, and S. T. Wu, "Tunable Fresnel lens using nanoscale polymer-stabilized liquid crystals," Appl. Phys. Lett. 83, 1515-1517 (2003).

12. Y. H. Fan, H. Ren, and S. T. Wu, "Electrically switchable Fresnel lens using a polymer-separated composite film," Opt. Express 13, 4141-4147 (2005).

13. J. Sun, S. Xu, H. Ren, and S. T. Wu, "Reconfigurable fabrication of scattering-free polymer network liquid crystal prism/ grating/lens," Appl. Phys. Lett. 102, 161106 (2013).

14. Y. H. Fan, H. Ren, and S. T. Wu, "Switchable Fresnel lens using polymer-stabilized liquid crystals," Opt. Express 11, 3080-3086 (2003).

15. H. Nemati, E. Mohajerani, A. Moheghi, M. B. Rad, and N. H. Nataj, "A simple holographic technic for fabricating a LC/polymer switchable Fresnel lens," EPL 87, 64001 (2009).

16. L. C. Lin, H. C. Jau, T. H. Lin, and A. Y. Fuh, "Highly efficient and polarization-independent Fresnel lens based on dyedoped liquid crystal," Opt. Express 15, 2900-2906 (2007).

17. K. T. Cheng, C. K. Liu, C. L. Ting, and A. Y. G. Fuh, "Electrically switchable and optically rewritable reflective Fresnel zone plate in dye-doped cholesteric liquid crystals," Opt. Express 15, 14078-14085 (2007).

18. Y. Li, Y. S. Yu, L. Guo, S. Z. Wu, C. Chen, L. G. Niu, A. W. Li, and H. Yang, "High efficiency multilevel phase-type Fresnel zone plates produced by two-photon polymerization of SU-8," J. Opt. 12, 035203 (2010).

19. T. H. Lin, Y. Huang, A. Y. G. Fuh, and S. T. Wu, "Polarization controllable Fresnel lens using dye-doped liquid crystals," Opt. Express 14, 2359-2364 (2006).

20. A. K. Srivastava and R. Manohar, "Guest-host mode ferroelectric liquid crystals," Liq. Cryst. 38, 183-190 (2011).

21. V. Chigrinov, V. Kozenkov, and H. S. Kwok, Photoalignment of Liquid Crystalline Materials: Physics and Applications (Wiley, 2008).

22. J. Sun, A. K. Srivastava, L. Wang, V. G. Chigrinov, and H. S. Kwok, "Optically tunable and rewritable diffraction grating with photoaligned liquid crystals," Opt. Lett. 38, 2342-2344 (2013).

23. W. Hu, A. K. Srivastava, X. Liang, X. W. Lin, J. T. Sun, G. Zhu, V. Chigrinov, and Y. Q. Lu, "Polarization independent liquid crystal gratings based on orthogonal photoalignments," Appl. Phys. Lett. 100, 111116 (2012).

24. K. C. Lo, J. D. Wang, and C. R. Lee, "Electrically controllable and polarization-independent Fresnel zone plate in a circularly symmetric hybrid-aligned liquid crystal film with a photoconductive polymer layer," Appl. Phys. Lett. 91, 181104 (2007). 